\title{
Current status and perspectives of interventional clinical trials for glioblastoma - analysis of ClinicalTrials.gov
}

\author{
Nikola Cihoric ${ }^{1 *+} \mathbb{D}$, Alexandros Tsikkinis $^{2 \dagger}$, Giuseppe Minniti ${ }^{3}$, Frank J Lagerwaard ${ }^{4}$, Ulrich Herrlinger ${ }^{5}$, \\ Etienne Mathier ${ }^{1}$, Ivan Soldatovic ${ }^{6}$, Branislav Jeremic ${ }^{7}$, Pirus Ghadjar ${ }^{8}$, Olgun Elicin ${ }^{1}$, Kristina Lössl ${ }^{1}$, \\ Daniel M Aebersold ${ }^{1}$, Claus Belka ${ }^{9,10}$, Evelyn Herrmann ${ }^{1}$ and Maximilian Niyazi, ${ }^{9,10}$
}

\begin{abstract}
The records of 208.777 (100\%) clinical trials registered at ClinicalTrials.gov were downloaded on the 19th of February 2016. Phase II and III trials including patients with glioblastoma were selected for further classification and analysis. Based on the disease settings, trials were classified into three groups: newly diagnosed glioblastoma, recurrent disease and trials with no differentiation according to disease setting. Furthermore, we categorized trials according to the experimental interventions, the primary sponsor, the source of financial support and trial design elements. Trends were evaluated using the autoregressive integrated moving average model. Two hundred sixteen (0.1\%) trials were selected for further analysis. Academic centers (investigator initiated trials) were recorded as primary sponsors in $56.9 \%$ of trials, followed by industry $25.9 \%$. Industry was the leading source of monetary support for the selected trials in $44.4 \%$, followed by $25 \%$ of trials with primarily academic financial support. The number of newly initiated trials between 2005 and 2015 shows a positive trend, mainly through an increase in phase II trials, whereas phase III trials show a negative trend. The vast majority of trials evaluate forms of different systemic treatments (91.2\%). In total, one hundred different molecular entities or biologicals were identified. Of those, $60 \%$ were involving drugs specifically designed for central nervous system malignancies. Trials that specifically address radiotherapy, surgery, imaging and other therapeutic or diagnostic methods appear to be rare. Current research in glioblastoma is mainly driven or sponsored by industry, academic medical oncologists and neuro-oncologists, with the majority of trials evaluating forms of systemic therapies. Few trials reach phase III. Imaging, radiation therapy and surgical procedures are underrepresented in current trials portfolios. Optimization in research portfolio for glioblastoma is needed.
\end{abstract}

Keywords: Glioblastoma, Clinicaltrials.gov, Interventional Clinical Trials

\section{Introduction}

Glioblastoma is the most common and aggressive primary parenchymal brain tumor [1,2]. The current standard of care is based on maximal safe surgical resection followed by concurrent chemoradiation (CTRT) with temozolomide followed by six months of maintenance chemotherapy, resulting in median survival rates of approximately 15 months [3]. Other than temozolomide, only few

\footnotetext{
* Correspondence: nikola.cihoric@gmail.com

${ }^{\dagger}$ Equal contributors

'Department of Radiation Oncology, Inselspital, Bern University Hospital, University of Bern, 3010 Bern, Switzerland

Full list of author information is available at the end of the article
}

agents have shown a clinical benefit to treatment with radiotherapy alone [4].

Furthermore, advanced immunotherapeutic strategies have also emerged, thus far without any significant success [5]. The current evidence-based treatment recommendations for systemic therapy are well summarized in the work from Olson et al. [6].

On review of among others recently reported ASTRO [7] and ESTRO-ACROP guidelines for glioblastomas [8], it would appear that substantial innovations with respect to radiotherapy approaches such as target definition, fractionation and planning as well as delivery techniques are largely lacking, or at least these innovations do not appear to find their way to current guidelines. From this 
unsubstantiated observation, the question arises whether these components of modern radiotherapy have been an integral part of past or current trials performed in glioblastoma patients.

In search of higher transparency and accessibility to information, several institutions and groups have established publicly available clinical trial registries. Trial registration is being regulated with European and US federal laws as well as international conventions (World Health Organization, WHO) $[9,10]$. Registration of all interventional clinical trials is obligatory in the European Union (EU) and the United States (US) and is required by an international consortium of medical journal editors [11]. ClinicalTrials.gov is the largest clinical trial registry with over 200,000 registered trials and a high weekly growth rate of new entries. The registration process and its potential for an in-depth analysis of the clinical trials' landscape is well described in the literature [12-16]. A detailed description of registered protocol elements can be found at the ClinicalTrials.gov website [17-19]. Due to the nature of ClinicalTrials.gov trial submission process, detailed information on past and present clinical trials can be obtained using the ClinicalTrials.gov registry; usually even more details than reported in the eventual peerreviewed publication.

The aim of the current study was to investigate in how far radiotherapy innovations, in any aspect, have been an integral part within the setup of past and current clinical trials. In recent years, an abundance of phase I trials have been initiated and completed, however, these trials generally have focused on the addition of systemic components in addition to standard forms of (radiotherapy or surgery) treatment, and not as much on potential innovations in the radiotherapy process. Therefore, we restricted the current analysis to phase II and III clinical trials for glioblastoma, reported in ClinicalTrials.gov in recent years.

\section{Materials and Methods Data acquisition}

The records of all 208,777 clinical trials registered at ClinicalTrials.gov were downloaded on the 19th of February 2016 and an SQL database was created to enable further analysis. The following fields were searched for glioblastoma-related keywords (glioblastoma, astrocytoma grade 4 (iv), gliosarcoma, gbm): short title, scientific title, conditions, a short summary and detailed description. Using this search strategy, a total of 1.064 trials were identified. We selected trials registered during the time period from January 2005 to December 2015 for further manual review. After exclusion of prematurely closed, phase I or observational trials and trials not specific for glioblastoma, 216 (20.3\%) trials were selected for analysis. The trial selection process is shown in Fig. 1.
Selected trials were divided into three groups: those concerning newly diagnosed glioblastoma, those in recurrent disease and finally trials where it was not specified or both categories were included.

All registered interventions were classified according to their specific role within the trial as part of the standard treatment or the experimental approach. Date of trial registration was taken into account. Systemic treatments were categorized based on the resources available on the following databases: www.drugbank.ca [20], National Cancer Institute Dictionary of Cancer Terms (www.cancer. gov), pubchem.ncbi.nlm.nih.gov as well as the Scopus database, the PubMed Database, Google Scholar and also through a generic internet search (Google search engine). We classified systemic treatments into experimental and approved drugs based on the development status of the specific therapy. Experimental drugs were annotated as such if no previous indication was approved for commercial usage from the United State Food and Drug Administration (FDA). The investigational compounds were further classified based on their specific chemical subgroups according to the World Health Organization ATC/DDD system $[21,22]$. By data unavailability, we manually classified the selected drugs according to their chemical group, as suggested in the WHO guideline [22]. Additionally, the specific target receptors and the mechanism of action were also searched and noted.

Radiotherapy, surgical procedures, and imaging procedures were classified according to their role in a clinical trial as part of the standard treatment or as an experimental intervention. If the procedure was in the focus of a particular trial it was considered as an experimental intervention. All surgical, as well as radiotherapy approaches in the recurrent setting, were considered experimental. Finally, all other interventions that do not belong to the mentioned groups, but were evaluated within a trial protocol were also considered as experimental.

In classifying the source of funding we used a modified strategy based on the methodology previously described in the work of Califf et al. [14].

\section{Statistical methodology}

Forecasting has been performed using the ARIMA (Autoregressive Integrated Moving Average) model. Based on Akaike information criterion (AIC), Rootmean-square deviation (RMSE) and R squared, confidence interval and logical outcome, the best model was chosen. The time interval was 11 years divided into quarters. We used the autoregressive order 1, difference 0 and moving average 1 [23]. Only non-seasonal structure was used. Forecasting was performed using R 3.3.0 statistical software. 


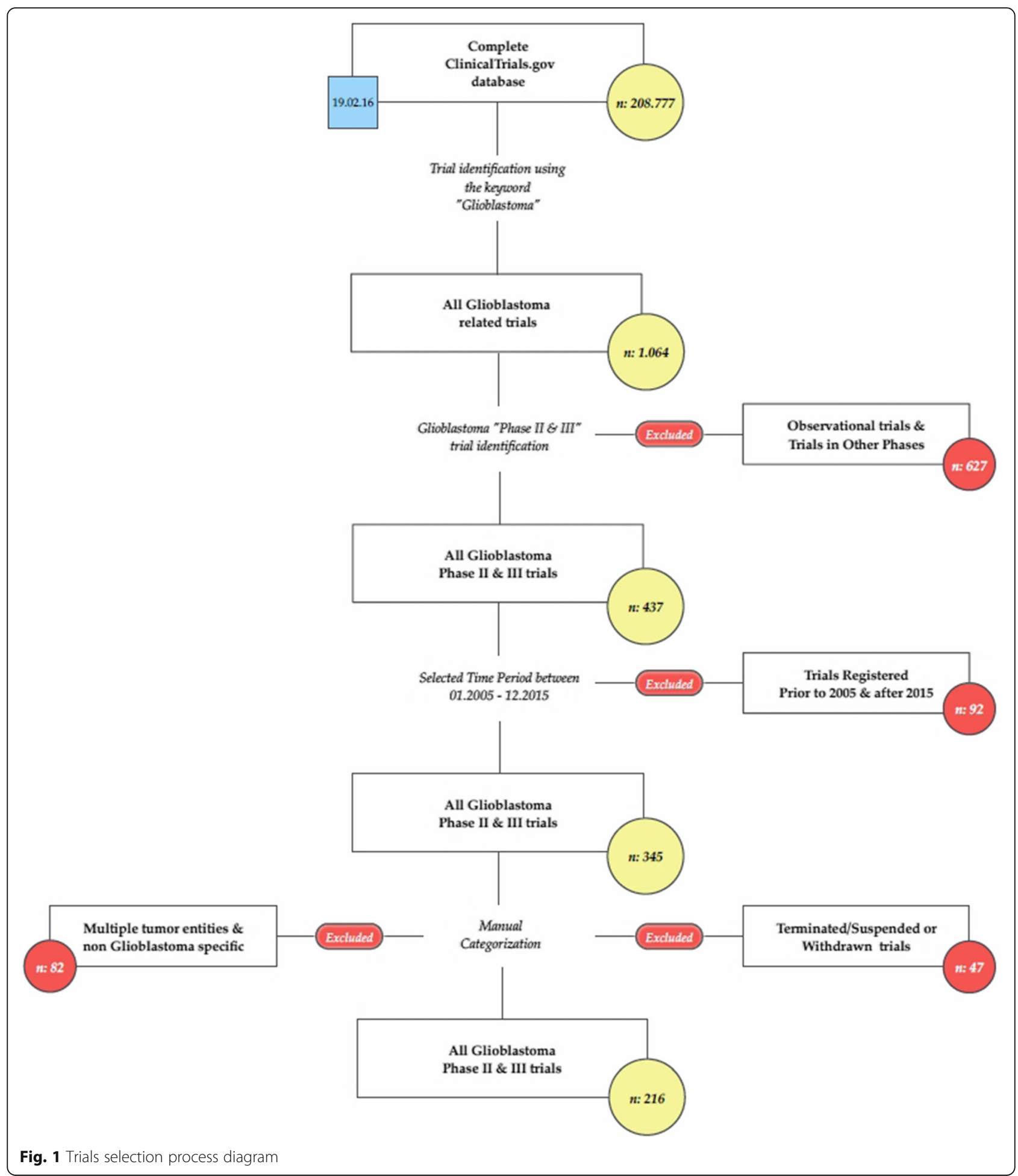

\section{Results}

Trial design characteristics are presented in Table 1 and a general overview of the trials is shown in Table 2 .

Academic centers (investigator-initiated trials) were recorded as primary sponsors in $56.9 \%$ of the trials, followed by industry $25.9 \%$. Industry is the leading source of monetary support in $44.4 \%$, followed by academia in $25 \%$. Other sponsors and sources of monetary support are presented in Fig. 2.

The number of yearly initiated trials between 2005 and 2015 ranged between 1 and 11, and shows a slightly positive trend (Fig. 3). 
Table 1 Trials design data

\begin{tabular}{|c|c|c|}
\hline & Number & Percent \\
\hline \multicolumn{3}{|l|}{ Trial Phase } \\
\hline Phase 2 & 188 & 87.0 \\
\hline Phase 2/Phase 3 & 3 & 1.4 \\
\hline Phase 3 & 25 & 11.6 \\
\hline \multicolumn{3}{|l|}{ Number of Arms } \\
\hline 1 & 107 & 49.5 \\
\hline 2 & 83 & 38.4 \\
\hline$\geq 3$ & 13 & 9.7 \\
\hline NR & 5 & 2.3 \\
\hline \multicolumn{3}{|l|}{ Sample Size } \\
\hline 0 to 50 & 90 & 41.7 \\
\hline 51 to 100 & 62 & 28.7 \\
\hline 101 to 200 & 36 & 16.7 \\
\hline 201 to 300 & 11 & 5.1 \\
\hline 301 or more & 16 & 7.4 \\
\hline NR & 1 & 0.5 \\
\hline \multicolumn{3}{|l|}{ Interventional Model } \\
\hline Single Group Assignment & 116 & 53.7 \\
\hline Parallel Assignment & 92 & 42.6 \\
\hline Factorial Assignment & 1 & 0.5 \\
\hline Crossover Assignment & 2 & 0.9 \\
\hline NR & 5 & 2.3 \\
\hline \multicolumn{3}{|l|}{ Treatment Allocation } \\
\hline Non-Randomized & 47 & 21.8 \\
\hline Randomized & 86 & 39.8 \\
\hline NR & 83 & 38.4 \\
\hline \multicolumn{3}{|l|}{ Masking (Blinding) } \\
\hline Open Label & 187 & 86.6 \\
\hline Single Blind & 2 & 0.9 \\
\hline Double Blind & 25 & 11.6 \\
\hline NR & 2 & 0.9 \\
\hline \multicolumn{3}{|l|}{ Endpoint Classification } \\
\hline Safety/Efficacy Study & 109 & 50.5 \\
\hline Efficacy Study & 75 & 34.7 \\
\hline Safety Study & 4 & 1.9 \\
\hline Bio-equivalence Study & 1 & 0.5 \\
\hline Pharmacodynamics Study & 1 & 0.5 \\
\hline Pharmacokinetics Study & 1 & 0.5 \\
\hline NR & 25 & 11.6 \\
\hline \multicolumn{3}{|l|}{ Primary Purpose of Trial } \\
\hline Treatment & 207 & 95.8 \\
\hline Diagnostic & 3 & 1.4 \\
\hline Health Services Research & 1 & 0.5 \\
\hline Basic Science & 2 & 0.9 \\
\hline Supportive Care & 1 & 0.5 \\
\hline NR & 2 & 0.9 \\
\hline
\end{tabular}

Table 2 Trial characteristics

\begin{tabular}{|c|c|c|}
\hline & Number & Percent \\
\hline \multicolumn{3}{|l|}{ Disease Settings } \\
\hline 1 & 5 & 2.3 \\
\hline 2 & 96 & 44.4 \\
\hline 3 & 115 & 53.2 \\
\hline \multicolumn{3}{|c|}{ Systemic Therapy as Investigative Intervention } \\
\hline 0 & 19 & 8.8 \\
\hline 1 & 95 & 44.0 \\
\hline 2 & 84 & 38.9 \\
\hline 3 & 17 & 7.9 \\
\hline 4 & 1 & 0.5 \\
\hline \multicolumn{3}{|c|}{ Radiotherapy as Investigative Intervention } \\
\hline Not Used & 121 & 56.0 \\
\hline Part of Standard Protocol & 75 & 34.7 \\
\hline Experimental & 20 & 9.3 \\
\hline \multicolumn{3}{|c|}{ Imaging as Investigative Intervention } \\
\hline Not Mentioned ${ }^{a}$ & 206 & 95.4 \\
\hline Experimental & 10 & 4.6 \\
\hline \multicolumn{3}{|c|}{ Surgery as Investigative Intervention } \\
\hline Not Used & 134 & 62.0 \\
\hline Part of Standard Protocol & 71 & 32.9 \\
\hline Experimental & 11 & 5.1 \\
\hline \multicolumn{3}{|l|}{ Trial Overall Status } \\
\hline Completed & 93 & 43.1 \\
\hline Active, not recruiting & 52 & 24.1 \\
\hline Not yet recruiting & 13 & 6.0 \\
\hline Recruiting & 58 & 26.9 \\
\hline \multicolumn{3}{|l|}{ Primary Sponsor Type } \\
\hline Industry & 56 & 25.9 \\
\hline $\mathrm{NIH}$ & 20 & 9.3 \\
\hline academy & 123 & 56.9 \\
\hline collaborative group & 17 & 7.9 \\
\hline \multicolumn{3}{|l|}{ Date of Registration } \\
\hline $2005-2009$ & 101 & 46.8 \\
\hline 2010-2015 & 115 & 53.2 \\
\hline
\end{tabular}

${ }^{a}$ Majority of trials do not mention imaging procedures in any contest. Registered Data do not provide possibility to extract information about utilization of imaging procedures as part of standard protocol

\section{Systemic therapy}

The majority of trials evaluate forms of systemic therapeutic approaches $(n=197,91.2 \%) .43 .7 \%$ of those were in primary, $53.8 \%$ in recurrent and $2.5 \%$ in both disease settings. Majority was phase II (87.8\%), followed by phase III (11.2\%). 54.3\% of trials on systemic therapy was single arm without adequate comparator. In total 100 different molecular agents or biologicals were identified. Of those, 40 (40\%) had been initially approved for 


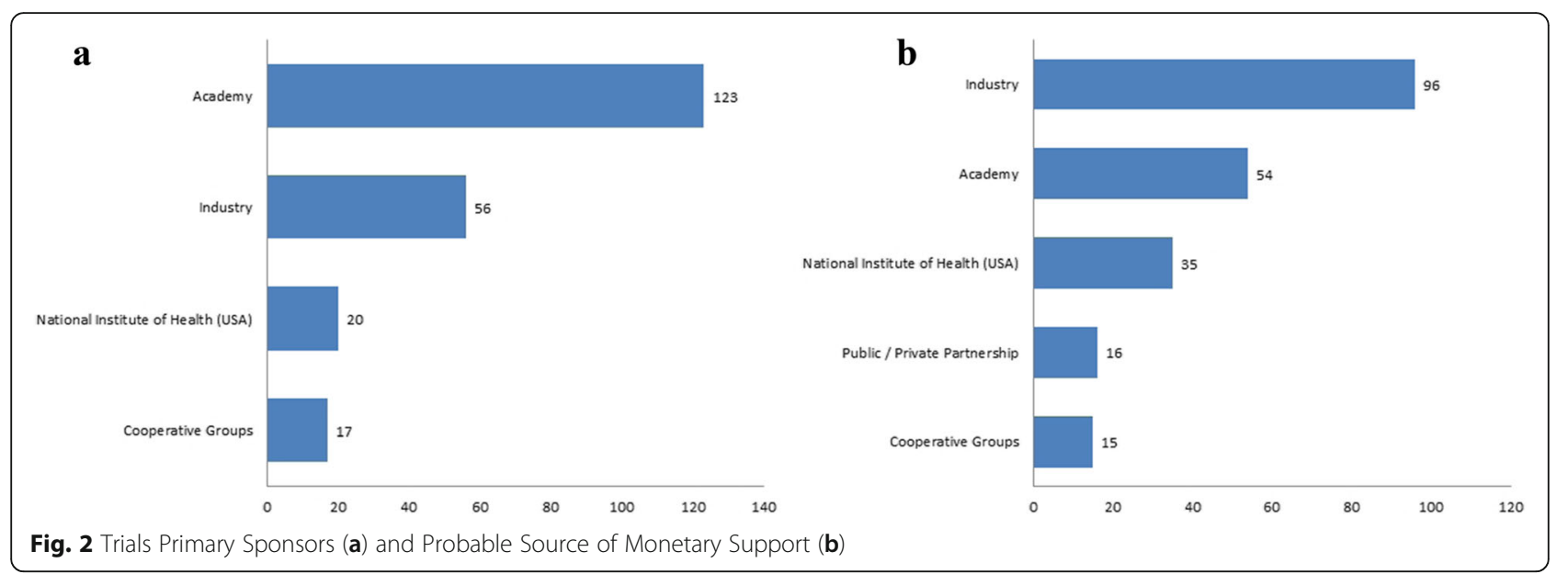

indications other than glioblastoma or CNS malignancies. Two (2\%) were approved for glioblastoma or other CNS or solid malignancies (carmustine, lomustine) and just one (1\%), namely temozolomide, for glioblastoma. Of the 57 (57\%) compounds with investigational status 37 (37\%) had been investigated for other tumors and one for non-malignant conditions (1\%). Nineteen (19\%) substances were developed specifically for the treatment of glioblastoma.

Classifying by drug type, 67 (67\%) substances belong to small molecules, 32 (32\%) to biologicals and for one (1\%) substance we did not find any data. 42 (42\%) compounds were registered in the WHO ACT/DDD database, with the most common group being protein kinase inhibitors $(n=14,14 \%)$, followed by "other neoplastic agents" $(n=7,7 \%)$, monoclonal antibodies $(n=5,5 \%)$ and lastly immunosuppressants and nitrosoureas (each represented with 2 compounds $(n=4,4 \%))$. The last 12 registered products belong to different classes.

The remaining 58 (58\%) compounds not registered in the WHO ACT/DDD database most commonly evaluated protein kinase inhibitors $(n=11,11 \%)$, followed by monoclonal antibodies $(n=13,13 \%)$ of which 5 were conjugated with toxins or radioisotopes and $6(6 \%)$ vaccines. Alkylating agents, DNA repair inhibitors, and other antineoplastic agents were each represented by 2 (4\%) substances. Twenty-two (22\%) substances belong to other individual classes.

For 30 systemic therapeutic entities, we did not find any specific target receptor or pathway. The most commonly researched targeted therapy aimed the EGFR receptor or its pathway $(n=11,11 \%)$, followed by VEGF $(n=8,8 \%)$ and multi-TKIs $(n=5,5 \%)$. An overview of

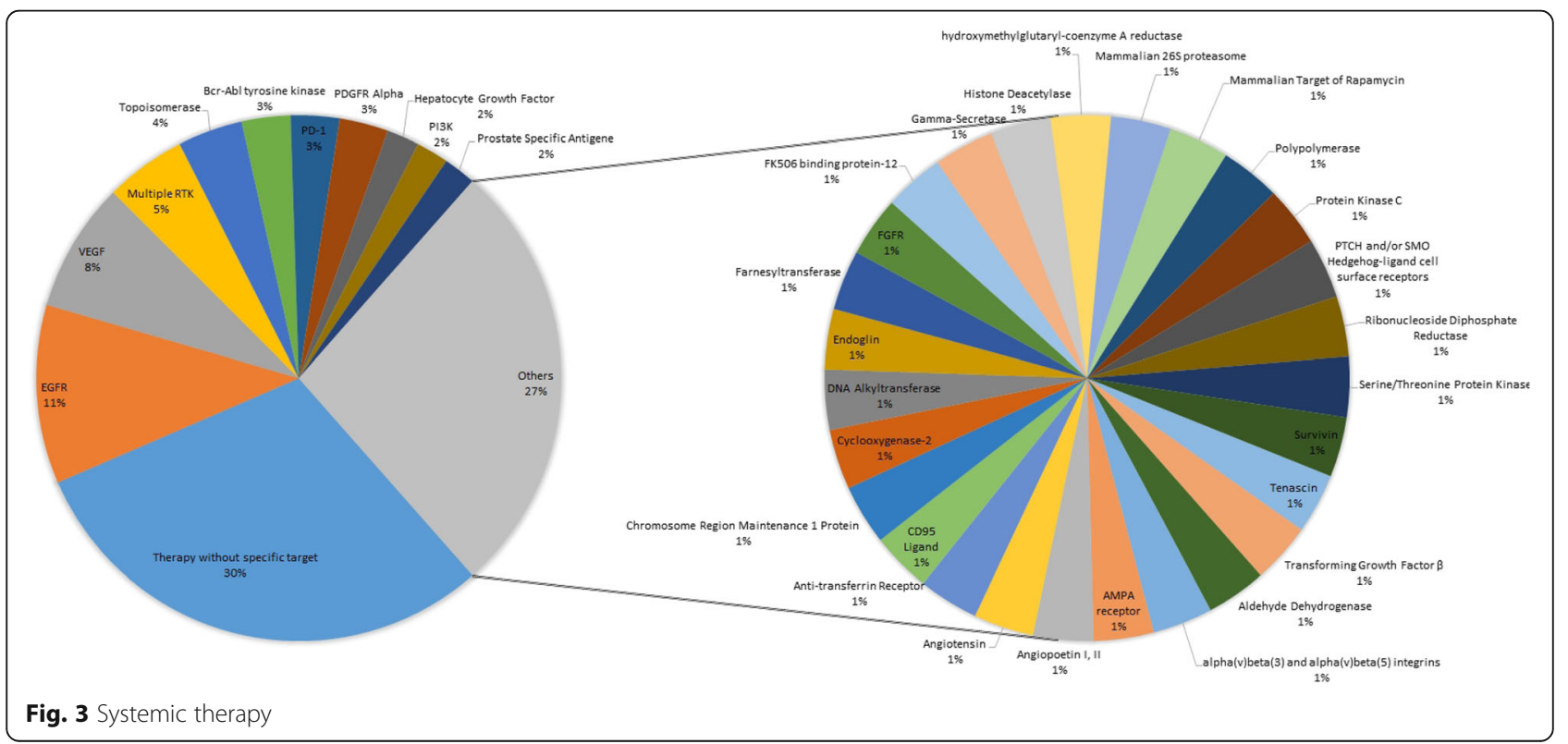


all the investigated systemic therapy agents is presented in Additional file 1: Appendix 2. and Fig. 3.

\section{Surgery}

Primary glioblastoma In the primary setting, surgery belonged to the investigational arm in four trials. Three of these studies reported on the use of 5-aminolevulinic acid (5-ALA) as a guidance help during surgery. One single other study concentrated, among other endpoints, on the cost effectiveness ratio between 5-ALA contrast enhanced surgery versus placebo.

Recurrent glioblastoma Only a single trial (NCT02394626 - RESURGE) concentrated solely on the efficacy of surgery. RESURGE is a randomized phase II trial with aim to evaluate value of surgical resection followed by second line therapy compared with second line therapy alone. The results should serve as a basis for larger phase III trial. In 6 other studies, surgery was part of the combined modality treatment for recurrent GBM in combination with chemotherapeutic strategies.

Radiotherapy Twenty trials ( $9.3 \%$ of all) evaluate RT as experimental interventions. Of those only one $(0.5 \%)$ was phase III, namely NCT01450449 trial, sponsored by the International Atomic Agency on short (5x5 Gy) vs. standard course (15x2.7 Gy) of RT in elderly patients.

Primary glioblastoma Fifteen $(6.9 \%$ of all) trials concentrated on radiotherapy in the primary setting, with four (1.9\%) trials exploring hypofractionated regimens, six (2.8\%) dose escalation, three (1.4\%) target volume definition (Subventricular zone RT, delineation with MRI vs. PET, Whole Brain Low Dose RT) with one (0.5\%) trial evaluating the Boron-Neutron Capture Therapy and one $(0.5 \%)$ comparing Intensity-modulated Radiotherapy (IMRT) versus Intensity-modulated Proton Therapy (IMPT).

Recurrent glioblastoma Five $(2.3 \%$ of all $)$ trials were evaluating the RT in the recurrent setting. Two (0.9\%) trials explored bevacizumab with or without radiotherapy where in one trial RT was applied in form of radiosurgery. One $(0.5 \%)$ combined APG-101 with re-irradiation with a dose of 36 Gy. One (0.5\%) evaluates two hypo-fractionated regimen ( $5 \times 5$ Gy vs $5 \times 7$ Gy), and one $(0.5 \%)$ evaluates delineation based on Amino-Acid PET with dose based on MRI.

\section{Imaging}

Six trials evaluate imaging procedures in the primary setting. Of those three evaluated MRI spectroscopy in delineating the radiotherapy volume and two FMISO-PET as a potential predictor. One trial directly compared MRI versus FDG-PET in target volume delineation. Four trials evaluated procedures in recurrent glioblastoma, one comparing MRI versus Amino acid-PET for target delineation, one FET-PET for evaluating the response to bevacizumab, one FMISO-PET, and MRI to evaluate the delivery of bevacizumab and one investigating the value of MRI for target delineation under bevacizumab treatment.

\section{Other investigative treatments}

Six trials explored the utilization of NovoTTF ${ }^{\circ}$ device, three Gliadel Wafer ${ }^{\circ}$, two transcranial magnetic stimulation, and one the local application of cellular adoptive immunotherapy (Autologous Lymphocytes). One trial explored the feasibility of molecular profiling with whole genome sequencing and one the treatment of anxiety in patients with glioblastoma.

The total number of trials initiated during the period (2005-2015) and forecast (2016-2020) is presented in Fig. 4. In regard to the several examined models, this model showed the optimal AIC (203.52) and RMSE (2.194). As presented in Fig. 2, in the next five years, it is expected that the number of studies show minor increase. Similarly with the previous model, increasing trend is observed regarding to number of phase II studies.

All trials evaluating surgery, radiotherapy, imaging and other investigative treatments are listed in Additional file 2: Appendix 1.

\section{Discussion}

In this work, we analyzed initiatives for trials for primary and recurrent glioblastoma in the last 10 year period. The total number of trials initiated yearly shows a slightly positive trend, although this appears to be mainly driven by a rise in the number of phase II trials, which constitute the vast majority of trials in primary and recurrent glioblastoma, whereby no difference exists in terms of disease setting (reccurent or primary). The most probable cause for this high initiation of trials will probably be the failure of previous early investigative treatments to show a clinical significant advantage. Rather than starting more trials unselectively, patients with (primary and recurrent) glioblastoma should be enrolled in early phase trials with novel designs, such as basket trials where patient selection is based on the genomic profile of the tumor rather than anatomical or classical histological classification [24].

Funding in glioblastoma reflects the situation across all clinical trials in oncology. Bradford et al. found that $41.8 \%$ of trials were funded primarily by the industry [25], which corresponds with the $51.9 \%$ in our study. A reason for concern should be the lack of industrial funding for research beyond forms of systemic therapy. The industry has displayed a strong interest in sponsoring trials for glioblastoma, and a variety of drugs are already in the investigative pipeline. At the same time, surgery, 

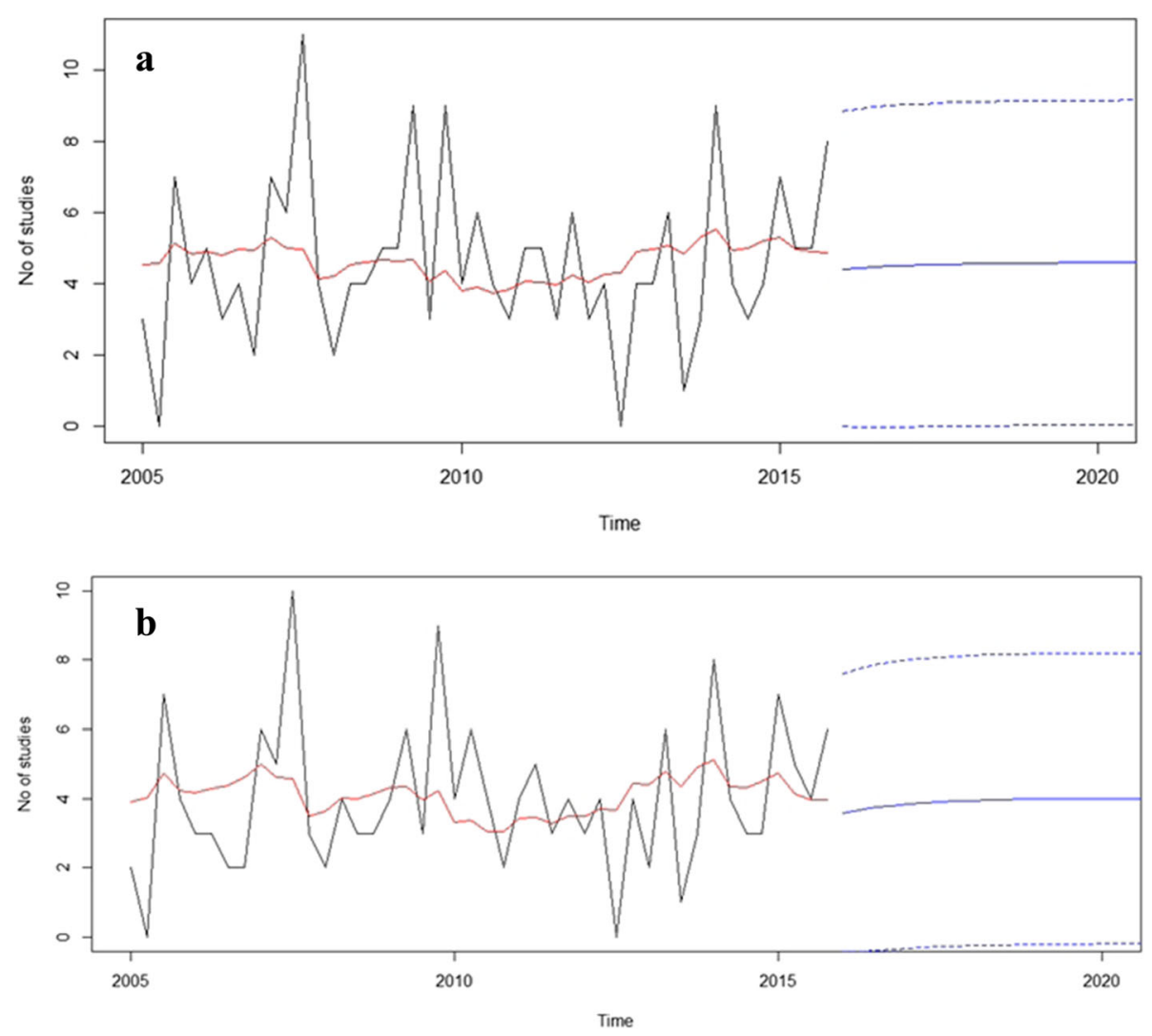

Fig. 4 New trials per year. Panel a shows ARIMA forecast model for all evaluated trials. Panel b shows ARIMA forecast model for phase II trials only

radiotherapy, and imaging focused trials represent just a small part of the glioblastoma clinical trial portfolio, being investigated in the experimental part in only $9.7 \%$ of all phase II/III glioblastoma trials. Research of the aforementioned modalities is supported and driven only from academic institutions while collaborative groups or NIH support is sparse.

It appears that even in the academic community either the interest, or the resources, for initiating of imaging, radiotherapy or surgery research are limited. Of the 123 trials initiated by academic centers, only a minority (19.5\% of all GBM academic trials) evaluated these treatment modalities in the experimental setting. Systemic therapies are the focus of investigation in $66 \%$ of purely academic trials, without registered or published external financial support. It appears that medical oncologists are leading the way in academic circles with innovative approaches in the treatment of glioblastoma. Based on the available data on ClinicalTrials.gov, we cannot assess whether the required research funding originates from third party sources such as research grants or private donations or from the academic institution.

Trials evaluating exclusively glioblastoma may appear rare when expressed in relative numbers $(0.1 \%$ of all registered trials). Nevertheless, it is worth mentioning that malignancies of the central nervous system represent a high percentage of the overall cancer trials portfolio and are more often in the research focus compared to other tumor entities with often significantly higher incidences, such as uterine, bladder and esophageal cancer [25].

Surgery in glioblastoma patients is of particular importance and should be evaluated in detail. Trials in the primary setting with surgery in focus, evaluate application of 5-ALA tissue contrast and only one trial evaluates resection optimization but from an economic perspective (NCT01811121). Keeping in mind the poor prognosis of glioblastoma and the predominance of failures at or near the original tumor location, the role of maximal safe surgical resection, lead to better outcome. There is solid data showing that the brain plasticity is more flexible than what was previously thought and therefore a more radical resection could be feasible [26]. The extent of resection is a known prognostic factor that influences survival. Lacroix at al. has shown that the extirpation of $98 \%$ or more of gadolinium-enhancing tumor mass was necessary to achieve significant survival [27] and Sanai et al. concluded that at least $78 \%$ of the enhancing tumor mass should be resected [28]. Finally, Marko et al. concluded in a recent analysis that any degree of resection is associated with a survival benefit [29]. All three analyses are based on 
retrospective data and this topic deserves a prospective evaluation. However, it seems that surgery does not belong to the research focus of individual researchers, industry or collaborative groups.

Next well established therapy for glioblastoma is radiation therapy, where the standard dose for primary GMB in younger patients with a good performance status is $60 \mathrm{~Gy}$ [3]. For elderly patients hypofractionated radiotherapy results in only a small benefit compared to the best supportive care [30], with the most important prognostic factor being age and the performance status [31].

Trials concentrated on specific radiotherapy questions are mostly in phase II, with a small sample size and initiated mainly through the academy.

As glioblastoma incidence is the highest in older patients, the optimal therapy for this particular group is of high interest. The currently available data suggest that surgical resection may play an important role [32, 33]. Adjuvant therapy is still a subject of investigation. Some data suggest that a combination therapy (RT and temozolomide) may cause excess toxicity [34,35] and the current evidence suggests that radiotherapy alone should be preferred in patients without MGMT promoter methylation (Methusalem NOA-8 trial). Hypofractionation is an attractive option for this particular patient group [36, 37]. In addition, there is initial evidence that hypofractionated radiotherapy may be combined with temozolomide and result in a better outcome without the excess toxicity [38, 39]. The NCT00482677 trial, could potentially provide definitive answers. Here hypofractionation alone was compared with hypofractionation and temozolomide in a population older than 65 years. The preliminary findings of this trial presented at ASCO 2016 showed a significant benefit for the combined therapy schedule.

Alternative radiotherapy schedules, dose or volume alterations could be of interest for the treatment of glioblastoma regardless of patient age [40], although we currently do not have high-quality prospective data. Moderate dose escalation trials with conventional radiotherapy methods failed to show an improvement in the outcome [41]. Irradiation of the gross tumor volume, even with higher doses, may be insufficient owed perhaps to the infiltrative nature of glioblastoma [42]. Higher dose escalation to the extended volume is not feasible with the standard techniques due to the close proximity of critical structures. The current body of knowledge in regard to the benefits of dose escalation is limited, but an intriguing report on dose escalation comes from Tsien et al. Patients were treated with 66-81 Gy delivered by IMRT with concurrent temozolomide. No grade 3 toxicities were reported and a median survival of 20.1 months was observed [43]. Several trials evaluated dose escalation, with one of them deserving particular mention: "MRSI Guided Dose Escalated Radiation in
Glioblastoma" (NCT02394665). Here investigators want to utilize the capabilities of magnetic resonance spectroscopy imaging to detect in addition to the standard MRI and high-risk area at risk of failure, the metabolically active areas in newly diagnosed glioblastoma. Patients will in a first phase undergo standard IMRT with an additional stereotactic radiosurgical boost to the High-Risk Tumor Volume as defined by 3D MRSI [44].

Furthermore, investigators try to evaluate volume alterations together with dose de-escalation in the two Phase II trials namely NCT01822275 and NCT02177578. The tumor location in regard to the Subventricular Zone seems to be of importance [45].

The combination of systemic therapies with radiation as a chemosensitizing agent is certainly attractive. A notable example is trial NCT01071837, where investigators combined re-irradiation (36 Gy/2 Gy ED) with a CD95 ligand inhibitor (APG101). Compared with re-irradiation alone, the combination with APG101 shows a better 6-month progression free survival and slightly better overall survival [46]. But radiosensitization is not a well-understood process and may be dependent on specific cell properties such as genetic alterations. Some tumor cell lines can be resistant to specific radiosensitizers depending on their molecular profile [47]. In addition, glioblastoma evolves during the time, and some passenger mutations may be responsible for resistance development, either towards chemo or radiotherapy.

Several trials are initiated in an effort to answer these questions. The influence of radiotherapy on the clinical course of the disease is certainly of the highest interest. However, there are additional factors, beyond toxicity and efficacy, that should be considered if radiotherapy is applied. Some speculated that radiotherapy may even increase the malignant potential of the tumors [48, 49]. This hypothesis seems to be especially valid for glioblastoma $[50,51]$. Accelerated repopulation or selection of more resistant cells is well recognized and described the process in many tumor entities, and it may be that the combination of geographical miss or an underdosing of the volume of interest (recognized or not) plays a role.

Imaging modalities are rarely evaluated in the prospective setting. The most commonly utilized experimental imaging modalities are MRI spectroscopy and FMISO-PET-CT. Besides, an FET-PET and Amino-Acid PET are used for the delineation of radiotherapy treatment volume in recurrent glioblastoma. Imaging is used to predict treatment response or to delineate the volume of interest. All but one trial (NCT01507506) are in phase II with low sample size. Further exploring of this area is necessary. Furthermore, the size and role of the optimal radiotherapy margins are also a matter of debate $[52,53]$. This is of particular importance in recurrent glioblastoma, where we have significantly less experience. Evaluation of 
different imaging modalities in prospective settings may play an important role in reducing this uncertainty [54]. Beside the target delineation, information gathered from different sources, such as MRI, may be used for dose calculations [55]. Furthermore, imaging is very interesting for the evaluation of disease progression and response to the therapy. It may be potentially used for the patient selection that will respond well to the systemic therapy $[56,57]$.

Although some research activity in imaging and radiotherapy is present, this is not enough, especially if compared with trials that evaluate systemic approaches. Current margins of $1.5-2 \mathrm{~cm}$ and a RT dose concept for GBM (60 Gy in 2 Gy fraction) are based on relapse pattern analyses and toxicity profiles from a pre-systemic era. If those are still necessary remains unclear and it will be hardly possible to change current practice on a larger scale without phase III data. However we did not detect any trials to address these specific questions.

The majority of evaluated systemic therapies target specific receptors or pathways. Beside protein kinase inhibitors, which represent the majority of evaluated drugs, we detected over 20 different substances that try to target other pathways and also 30 substances that do not aim a specific pathway, mostly cytotoxic agents. Interesting findings of this study is that $57 \%$ of the investigated substances were not approved for marketing in any tumor entities, but only $24 \%$ were initially intended for use in glioblastoma or CNS malignancies. As the majority of those are sponsored or supported by the industry, this indicates that glioblastoma is an attractive target even financially. A small benefit in any of the endpoints may provide significant marketing advantages and possibly profit, even when accounting the low overall incidence of glioblastoma. Currently, there is only two systemic therapies approved for glioblastoma two from the cytotoxic group and one from the targeted group. Glioblastoma is also an attractive target from a clinical trial workflow perspective. Short term follow-up is certainly reducing the overall cost of the trial [58].

It seems that immunotherapy is a promising treatment modality and the combination with other options could potentially have a synergistic effect. The potential of other therapy forms to induce an immune response is also recognized. One important preclinical study has already shown that fractionated radiation may induce cell death [59]. This should be evaluated further in order to better understand the complex processes between the immune system and CNS and tumor interactions.

Nevertheless results of phase II trials should always be regarded cautiously. The problem of promising therapies with consequent failures in pivotal studies is recognized. An improvement of the trial design would be beneficial to all involved parties [60].

At the time of analysis, the majority of trials were completed with $43.1 \%(n=93)$ trials reported as completed and $24.1 \%(n=52)$ with achieved recruitment goals. Even though the results for 39 (18.1\%) trials were reported, only 16 of them were accompanied by their publication on ClinicalTrials.gov. This could be attributed to several possible causes: Some of the published work was not captured by an automatic search of the PubMed database [61]. The second reason is the time required from finishing a trial to final publication, in some cases exceeding 30 months [62]. Some trials will also never reach publication for unknown reasons [63], with a significant proportion possibly attributed to negative result and as a result a lack of interest either by the editors or the authors.

\section{Limitations}

The presented analysis is not without its limitations. Some data might have been incorrectly registered in the ClinicalTrials.gov. Moreover, we cannot exclude the possibility that certain data were misclassified during the selection and classification. In addition, the data sets for all trials in the database are not always complete and up-to-date. However, we took great care to minimize these limitations: two authors (NC and AT) crosschecked all trials identified and the trial selection steps.

Additionally, there may be a bias in in terms of number of registered trials in recent years compared with early period. We believe that our analysis is unique and important and that it provides researchers and clinicians with a realistic picture of the future of glioblastoma treatment.

\section{Conclusions}

Investigation in glioblastoma is mainly driven or sponsored by the industry and medical oncologists, with the majority of trials evaluating forms of systemic therapies. Most of the trials are in phase II with just a few trials ever reaching phase III. Imaging, surgery and radiation therapy are heavily underrepresented treatment methods in terms of investigations. Optimization in research portfolio for glioblastoma is needed.

\section{Additional files}

Additional file 1: Appendix 2. Systemic agents investigated for treatment of glioblastoma. (DOCX $19 \mathrm{~kb})$

Additional file 2: Appendix 1. Trials that evaluate surgery,

radiotherapy, imaging and other treatment modalities. (DOCX $23 \mathrm{~kb}$ )

\section{Abbreviations}

5-ALA: 5-aminolevulinic acid; ADC: Apparent diffusion coefficient; AGT: O6alkylguanine-DNA alkyltransferase; AIC: Akaike information criterion; ATC Classification system: Anatomical Therapeutic Chemical classification system; BBB: Blood-brain Barrier; BNCT: Boron neutron capture therapy; Cl: Confidence Interval; CNS: Central Nervous System; CR: Complete Response; CSF: Cerebral Spinal Fluid; CTCAE: Common Terminology Criteria for Adverse Events; DCE: Dynamic Contrast-Enhanced; DCs: Dendritic cells; DD: Dose-dense; DDD: Defined Daily Dose; DI: Dose Intensified; DNA: Deoxyribonucleic acid; ECOG: Eastern Cooperative Oncology Group; EGFR: Epidermal growth factor receptor; EIAEDs: CYP3-A inducing anti-epileptics; EORTC: European Organisation 
for Research and Treatment of Cancer; EU: European Union; FDA: Food and Drug Administration; FDG: Fluorodeoxyglucose; FET: Fluoro-ethyl-tyrosin; Flt-3: Fms-like tyrosine kinase 3; FMISO: Fluoro misonidazole; GBM: Glioblastoma; GCIMP: Glioma-CpG island methylator phenotype; Gy: Gray; HR: Hazard ratio; IMPT: Intensity-modulated Proton Therapy; IMRT: Intensity-mModulated Radiotherapy; KLH: Keyhole limpet hemocyanin; KPS: Karnofsky Performance Status; LAK: Lymphokine-activated killer; MGMT: Methyl Guanine Methyl Transferase; MMP: matrix metalloproteinase; MRI: Magnetic Resonance Imaging; mRNA: Messenger ribonucleic acid; NCF: Neurocognitive function; NCl: National Cancer Institute; NCl: National Cancer Institute; O6-BG: O6-benzylguanine; OS: Overall Survival; PAKT: Phosphorylated Protein Kinase Strain; PDGFR: Platelet-derived growth factor receptors; PET: Positron emission tomography; PFS: Progression-free survival; Poly-ICLC: Poly-l-lysine and carboxymethylcellulose; PR: Partial Response; PTEN: Phosphatase and tensin homolog; QOL: Quality of life; RMSE: Root-mean-square deviation; RPA: Recursive partitioning analysis; RT: Radiotherapy; RTOG: Radiation Therapy Oncology Group; STD: Standard Dose; TKI: Tyrosine Kinase Inhibitor; TTP: Time to progression; US: United States; VEGF: Vascular endothelial growth factor; VEGFR: Vascular endothelial growth factor receptor; WHO: World Health Organization

\section{Acknowledgements}

We would like to thank Branislav Trninic and Tomislav Cuk from KapitalSoft Trebinje for technical support and advice for data processing.

\section{Funding}

No funding source to report.

\section{Availability of data and materials}

Basic dataset used for the review can be downloaded from following address: http://clinicaltrial.co/SharedData

http://clinicaltrial.co/Content/files/Basic\%20Data\%20for\%20Glioblastoma\%20 Review.xlsx

\section{Authors' contributions}

NC and MN developed study concept. NC has created initial database. NC together with AT and EM manually classified the data. IS has calculated statistics. All authors discussed the results and gave intellectual input sufficient for co-authorship. NC, AT and MN drafted the initial version of a manuscript. All other authors read and contributed to the final version. All authors read and approved the final version of the manuscript.

\section{Competing interests}

Nikola Cihoric is the owner of www.clinicaltrial.co domain. www.clinicaltrial.co is currently under development. www.clinicaltrial.co is a non-profit, free access, meta-research portal aimed to provide advanced text mining and data processing capability based on, but not limited to, open clinical trials registries. The other authors have no conflicts of interest to declare.

\section{Consent for publication}

Not applicable.

\section{Ethics approval and consent to participate}

Not applicable.

\section{Author details}

'Department of Radiation Oncology, Inselspital, Bern University Hospital, University of Bern, 3010 Bern, Switzerland. ²Department of Genitourinary Oncology, David H. Koch Center for Applied Research of Genitourinary Cancers, The University of Texas MD Anderson Cancer Center, Houston, Texas, USA. ${ }^{3}$ Unit of Radiation Oncology, Sant' Andrea Hospital, University Sapienza, and IRCCS Neuromed, Pozzilli (IS), Italy. ${ }^{4}$ Department of Radiation Oncology, VU University Medical Center, Amsterdam, The Netherlands. ${ }^{5}$ Department of Neurology, Division of Clinical Neurooncology, University of Bonn Medical Center, Bonn, Germany. ${ }^{6}$ Faculty of Medicine, University of Belgrade, Belgrade, Serbia. 'Institute of Lung Diseases, Sremska Kamenica, Serbia and BiolRC Center for Biomedical Research, Kragujevac, Serbia. ${ }^{8}$ Department of Radiation Oncology, Charité Universitätsmedizin Berlin, Berlin, Germany. ${ }^{9}$ Department of Radiation Oncology, LMU Munich, München, Germany. ${ }^{10}$ German Cancer Consortium (DKTK) \& German Cancer Research Center (DKFZ), Heidelberg, Germany.
Received: 23 September 2016 Accepted: 8 December 2016 Published online: 03 January 2017

\section{References}

1. Buckner JC. Factors influencing survival in high-grade gliomas. Semin Oncol. 2003;30(6 Suppl 19):10-4.

2. Curran Jr WJ, Scott CB, Horton J, Nelson JS, Weinstein AS, Fischbach AJ, Chang $\mathrm{CH}$, Rotman M, Asbell SO, Krisch RE, et al. Recursive partitioning analysis of prognostic factors in three Radiation Therapy Oncology Group malignant glioma trials. J Natl Cancer Inst. 1993;85(9):704-10.

3. Stupp R, Mason WP, van den Bent MJ, Weller M, Fisher B, Taphoorn MJ, Belanger K, Brandes AA, Marosi C, Bogdahn U, et al. Radiotherapy plus concomitant and adjuvant temozolomide for glioblastoma. N Engl J Med. 2005;352(10):987-96.

4. Burdett S, Pignon JP, Tierney J, Tribodet H, Stewart L, Le Pechoux C, Auperin A, Le Chevalier T, Stephens RJ, Arriagada R, et al. Adjuvant chemotherapy for resected early-stage non-small cell lung cancer. Cochrane Database Syst Rev. 2015;3:CD011430

5. Wollmann G, Ozduman K, van den Pol AN. Oncolytic virus therapy for glioblastoma multiforme: concepts and candidates. Cancer J. 2012;18(1):69-81.

6. Olson JJ, Nayak L, Ormond DR, Wen PY, Kalkanis SN, Committee ACJG. The role of cytotoxic chemotherapy in the management of progressive glioblastoma : a systematic review and evidence-based clinical practice guideline. J Neuro-Oncol. 2014;118(3):501-55.

7. Cabrera AR, Kirkpatrick JP, Fiveash JB, Shih HA, Koay EJ, Lutz S, Petit J, Chao ST, Brown PD, Vogelbaum M, et al. Radiation therapy for glioblastoma: Executive summary of an American Society for Radiation Oncology Evidence-Based Clinical Practice Guideline. Pract Radiat Oncol. 2016;6(4):217-25.

8. Niyazi M, Brada M, Chalmers AJ, Combs SE, Erridge SC, Fiorentino A, Grosu AL, Lagerwaard FJ, Minniti G, Mirimanoff RO, et al. ESTRO-ACROP guideline "target delineation of glioblastomas". Radiother Oncol. 2016;118(1):35-42.

9. WHO: WHO Trial Search Portal. In.

10. WMA. In: WMA Declaration of Helsinki. http://www.wma.net/en/ 30publications/10policies/b3/. Accessed 2 Nov 2016.

11. ICMJE: Clinical Trial Registration Statement. In.

12. Becker JE, Ross JS. Reporting discrepancies between the clinicaltrials.gov results database and peer-reviewed publications. Ann Intern Med. 2014; 161(10):760.

13. Cihoric N, Tsikkinis A, van Rhoon G, Crezee H, Aebersold DM, Bodis S, Beck M, Nadobny J, Budach V, Wust P et al.: Hyperthermia-related clinical trials on cancer treatment within the ClinicalTrials.gov registry. Int J Hyperthermia 2015;31:1-6.

14. Califf RM, Zarin DA, Kramer JM, Sherman RE, Aberle LH, Tasneem A. Characteristics of clinical trials registered in ClinicalTrials.gov 2007-2010. JAMA. 2012;307(17):1838-47.

15. Tsikkinis A, Cihoric N, Giannarini G, Hinz S, Briganti A, Wust P, Ost P, Ploussard G, Massard C, Surcel Cl et al.: Clinical perspectives from Randomized Phase 3 trials on Prostate Cancer: An Analysis of the Clinicaltrials.gov Database. European Urology Focus 2015.

16. Cihoric N, Tsikkinis A, Miguelez CG, Strnad V, Soldatovic I, Ghadjar P, Jeremic B, Dal Pra A, Aebersold DM, Lossl K. Portfolio of prospective clinical trials including brachytherapy: an analysis of the ClinicalTrials.gov database. Radiat Oncol. 2016;11(1):48. doi:10.1186/s13014-016-0624-8.

17. Gillen JE, Tse T, Ide NC, McCray AT. Design, implementation and management of a web-based data entry system for ClinicalTrials.gov. Stud Health Technol Inform. 2004;107(Pt 2):1466-70.

18. Tse T, Williams RJ, Zarin DA. Update on Registration of Clinical Trials in ClinicalTrials.gov. Chest. 2009;136(1):304-5.

19. Clarke JL, Molinaro AM, Phillips JJ, Butowski NA, Chang SM, Perry A, Costello JF, DeSilva AA, Rabbitt JE, Prados MD. A single-institution phase II trial of radiation, temozolomide, erlotinib, and bevacizumab for initial treatment of glioblastoma. Neuro Oncol. 2014;16(7):984-90.

20. Wishart DS, Knox C, Guo AC, Shrivastava S, Hassanali M, Stothard P, Chang Z, Woolsey J. DrugBank: a comprehensive resource for in silico drug discovery and exploration. Nucleic Acids Res. 2006;34(Database issue):D668-672.

21. Hjalgrim LL, Rostgaard K, Engholm G, Pukkala E, Johannesen TB, Olafsdottir E, Hjalgrim $\mathrm{H}$. Aetiologic heterogeneity in pediatric Hodgkin lymphoma? Evidence from the Nordic countries, 1978-2010. Acta Oncol. 2016;55(1):85-90.

22. WHO Collaborating Centre for Drug Statistics Methodology O, Norway. WHO Collaborating Centre for Drug Statistics Methodology, Guidelines for 
ATC classification and DDD assignment 2015. 19th ed. Oslo: World Health Organization Oslo; 2015

23. Brockwell PJ, Davis RA: Introduction to time series and forecasting: Springer Science \& Business Media; 2006

24. Redig AJ, Jänne PA. Basket trials and the evolution of clinical trial design in an era of genomic medicine. J Clin Oncol. 2015;33(9):975-7.

25. Hirsch BR, Califf RM, Cheng SK, Tasneem A, Horton J, Chiswell K, Schulman KA, Dilts DM, Abernethy AP. Characteristics of oncology clinical trials: insights from a systematic analysis of ClinicalTrials.gov. JAMA Intern Med. 2013;173(11):972-9.

26. Duffau H. Long-term outcomes after supratotal resection of diffuse lowgrade gliomas: a consecutive series with 11-year follow-up. Acta Neurochir (Wien). 2016;158(1):51-8.

27. Lacroix M, Abi-Said D, Fourney DR, Gokaslan ZL, Shi W, DeMonte F, Lang FF, McCutcheon IE, Hassenbusch SJ, Holland E, et al. A multivariate analysis of 416 patients with glioblastoma multiforme: prognosis, extent of resection, and survival. J Neurosurg. 2001;95(2):190-8.

28. Sanai N, Polley MY, McDermott MW, Parsa AT, Berger MS. An extent of resection threshold for newly diagnosed glioblastomas. J Neurosurg. 2011;115(1):3-8.

29. Marko NF, Weil RJ, Schroeder JL, Lang FF, Suki D, Sawaya RE. Extent of resection of glioblastoma revisited: personalized survival modeling facilitates more accurate survival prediction and supports a maximum-safe-resection approach to surgery. J Clin Oncol. 2014;32(8):774-82.

30. Keime-Guibert F, Chinot O, Taillandier L, Cartalat-Carel S, Frenay M, Kantor G, Guillamo JS, Jadaud E, Colin P, Bondiau PY, et al. Radiotherapy for glioblastoma in the elderly. N Engl J Med. 2007;356(15):1527-35

31. Scott JG, Bauchet L, Fraum TJ, Nayak L, Cooper AR, Chao ST, Suh JH, Vogelbaum MA, Peereboom DM, Zouaoui S, et al. Recursive partitioning analysis of prognostic factors for glioblastoma patients aged 70 years or older. Cancer. 2012;1 18(22):5595-600.

32. Vuorinen V, Hinkka S, Farkkila M, Jaaskelainen J. Debulking or biopsy of malignant glioma in elderly people - a randomised study. Acta Neurochir (Wien). 2003;145(1):5-10.

33. Almenawer SA, Badhiwala JH, Alhazzani W, Greenspoon J, Farrokhyar F, Yarascavitch B, Algird A, Kachur E, Cenic A, Sharieff W, et al. Biopsy versus partial versus gross total resection in older patients with high-grade glioma: a systematic review and meta-analysis. Neuro Oncol. 2015;17(6):868-81.

34. Minniti G, De Sanctis V, Muni R, Filippone F, Bozzao A, Valeriani M, Osti MF, De Paula U, Lanzetta G, Tombolini V, et al. Radiotherapy plus concomitant and adjuvant temozolomide for glioblastoma in elderly patients. J Neurooncol. 2008;88(1):97-103.

35. Brandes AA, Franceschi E, Tosoni A, Benevento F, Scopece L, Mazzocchi V, Bacci A, Agati R, Calbucci F, Ermani M. Temozolomide concomitant and adjuvant to radiotherapy in elderly patients with glioblastoma: correlation with MGMT promoter methylation status. Cancer. 2009; 115(15):3512-8.

36. Roa W, Brasher PM, Bauman G, Anthes M, Bruera E, Chan A, Fisher B, Fulton D, Gulavita S, Hao C, et al. Abbreviated course of radiation therapy in older patients with glioblastoma multiforme: a prospective randomized clinical trial. J Clin Oncol. 2004;22(9):1583-8.

37. Malmstrom A, Gronberg BH, Marosi C, Stupp R, Frappaz D, Schultz H, Abacioglu U, Tavelin B, Lhermitte B, Hegi ME, et al. Temozolomide versus standard 6-week radiotherapy versus hypofractionated radiotherapy in patients older than 60 years with glioblastoma: the Nordic randomised, phase 3 trial. Lancet Oncol. 2012;13(9):916-26.

38. Fiorentino A, Balducci M, De Bonis P, Chiesa S, De Filippo L, Mangiola A, De Rose F, Autorino R, Rinaldi C, Fersino S, et al. Can elderly patients with newly diagnosed glioblastoma be enrolled in radiochemotherapy trials? Am J Clin Oncol. 2015:38(1):23-7.

39. Minniti G, Lanzetta G, Scaringi C, Caporello P, Salvati M, Arcella A, De Sanctis V, Giangaspero F, Enrici RM. Phase II study of short-course radiotherapy plus concomitant and adjuvant temozolomide in elderly patients with glioblastoma. Int J Radiat Oncol Biol Phys. 2012;83(1):93-9.

40. Azoulay M, Santos F, Souhami L, Panet-Raymond V, Petrecca K, Owen S, Guiot MC, Patyka M, Sabri S, Shenouda G, et al. Comparison of radiation regimens in the treatment of Glioblastoma multiforme: results from a single institution. Radiat Oncol. 2015;10:106.

41. Badiyan SN, Markovina S, Simpson JR, Robinson CG, DeWees T, Tran DD, Linette G, Jalalizadeh R, Dacey R, Rich KM, et al. Radiation therapy dose escalation for glioblastoma multiforme in the era of temozolomide. Int $J$ Radiat Oncol Biol Phys. 2014;90(4):877-85.
42. Halperin EC, Bentel G, Heinz ER, Burger PC. Radiation therapy treatment planning in supratentorial glioblastoma multiforme: an analysis based on post mortem topographic anatomy with CT correlations. Int J Radiat Oncol Biol Phys. 1989;17(6):1347-50.

43. Tsien Cl, Brown D, Normolle D, Schipper M, Piert M, Junck L, Heth J, GomezHassan D, Ten Haken RK, Chenevert T, et al. Concurrent temozolomide and dose-escalated intensity-modulated radiation therapy in newly diagnosed glioblastoma. Clin Cancer Res. 2012;18(1):273-9.

44. Bodensohn R, Sohn M, Ganswindt U, Schupp G, Nachbichler SB, Schnell O, Belka C, Niyazi M. Hippocampal EUD in primarily irradiated glioblastoma patients. Radiat Oncol. 2014;9:276.

45. Adeberg S, Bostel T, Konig L, Welzel T, Debus J, Combs SE. A comparison of long-term survivors and short-term survivors with glioblastoma, subventricular zone involvement: a predictive factor for survival? Radiat Oncol. 2014;9:95.

46. Wick W, Fricke H, Junge K, Kobyakov G, Martens T, Heese O, Wiestler B, Schliesser MG, von Deimling A, Pichler J, et al. A phase II, randomized, study of weekly APG101 + reirradiation versus reirradiation in progressive glioblastoma. Clin Cancer Res. 2014;20(24):6304-13.

47. Kuger S, Flentje M, Djuzenova CS. Simultaneous perturbation of the MAPK and the PI3K/mTOR pathways does not lead to increased radiosensitization. Radiat Oncol. 2015;10:214.

48. Qian LW, Mizumoto K, Urashima T, Nagai E, Maehara N, Sato N, Nakajima M, Tanaka M. Radiation-induced increase in invasive potential of human pancreatic cancer cells and its blockade by a matrix metalloproteinase inhibitor, CGS27023. Clinical Cancer Res. 2002;8(4):1223-7.

49. Camphausen K, Moses MA, Beecken WD, Khan MK, Folkman J, O'Reilly MS Radiation therapy to a primary tumor accelerates metastatic growth in mice. Cancer Res. 2001;61(5):2207-11.

50. Cordes N, Hansmeier B, Beinke C, Meineke V, van Beuningen D. Irradiation differentially affects substratum-dependent survival, adhesion, and invasion of glioblastoma cell lines. Br J Cancer. 2003;89(11):2122-32.

51. Wild-Bode C, Weller M, Rimner A, Dichgans J, Wick W. Sublethal irradiation promotes migration and invasiveness of glioma cells: implications for radiotherapy of human glioblastoma. Cancer Res. 2001;61(6):2744-50.

52. Gebhardt BJ, Dobelbower MC, Ennis WH, Bag AK, Markert JM, Fiveash JB. Patterns of failure for glioblastoma multiforme following limited-margin radiation and concurrent temozolomide. Radiat Oncol. 2014;9:130.

53. Ali AN, Ogunleye T, Hardy CW, Shu HK, Curran WJ, Crocker IR. Improved hippocampal dose with reduced margin radiotherapy for glioblastoma multiforme. Radiat Oncol. 2014:9:20.

54. Miwa K, Matsuo M, Ogawa S, Shinoda J, Yokoyama K, Yamada J, Yano H, Iwama T. Re-irradiation of recurrent glioblastoma multiforme using 11C-methionine PET/ CT/MRI image fusion for hypofractionated stereotactic radiotherapy by intensity modulated radiation therapy. Radiat Oncol. 2014;9:181.

55. Jonsson JH, Akhtari MM, Karlsson MG, Johansson A, Asklund T, Nyholm T. Accuracy of inverse treatment planning on substitute $C T$ images derived from MR data for brain lesions. Radiat Oncol. 2015;10:13.

56. Lu-Emerson C, Duda DG, Emblem KE, Taylor JW, Gerstner ER, Loeffler JS, Batchelor TT, Jain RK. Lessons from anti-vascular endothelial growth factor and anti-vascular endothelial growth factor receptor trials in patients with glioblastoma. J Clin Oncol. 2015;33(10):1197-213.

57. Wen PY, Macdonald DR, Reardon DA, Cloughesy TF, Sorensen AG, Galanis E, Degroot J, Wick W, Gilbert MR, Lassman AB, et al. Updated response assessment criteria for high-grade gliomas: response assessment in neuro-oncology working group. J Clin Oncol. 2010;28(11): 1963-72.

58. Raftery J, Young A, Stanton L, Milne R, Cook A, Turner D, Davidson P. Clinical trial metadata: defining and extracting metadata on the design, conduct, results and costs of 125 randomised clinical trials funded by the National Institute for Health Research Health Technology Assessment programme. Health Technol Assess. 2015;19(11):1-138.

59. Rubner Y, Muth C, Strnad A, Derer A, Sieber R, Buslei R, Frey B, Fietkau R, Gaipl US. Fractionated radiotherapy is the main stimulus for the induction of cell death and of Hsp70 release of p53 mutated glioblastoma cell lines. Radiat Oncol. 2014;9(1):89.

60. Galanis E, Wu W, Cloughesy T, Lamborn K, Mann B, Wen PY, Reardon DA, Wick W, Macdonald D, Armstrong TS, et al. Phase 2 trial design in neurooncology revisited: a report from the RANO group. Lancet Oncol. 2012; 13(5):e196-204.

61. Huser V, Cimino JJ. Precision and negative predictive value of links between ClinicalTrials.gov and PubMed. AMIA Annu Symp Proc. 2012;2012:400-8. 
62. Ross JS, Tse T, Zarin DA, Xu H, Zhou L, Krumholz HM. Publication of NIH funded trials registered in ClinicalTrials.gov: cross sectional analysis. BMJ. 2012;344:d7292.

63. Jones CW, Handler L, Crowell KE, Keil LG, Weaver MA, Platts-Mills TF. Non-publication of large randomized clinical trials: cross sectional analysis. BMJ. 2013;347:f6104.

Submit your next manuscript to BioMed Central and we will help you at every step:

- We accept pre-submission inquiries

- Our selector tool helps you to find the most relevant journal

- We provide round the clock customer support

- Convenient online submission

- Thorough peer review

- Inclusion in PubMed and all major indexing services

- Maximum visibility for your research

Submit your manuscript at www.biomedcentral.com/submit 